\title{
Mini-partial lateral corpectomy and hemilaminectomy for the treatment of heavily protruded thoracolumbar intervertebral disc in small dogs
}

\author{
Hyun-Jung Han ${ }^{1}$, Hun-Young Yoon ${ }^{2, *}$ \\ ${ }^{1}$ Department of Veterinary Emergency Medicine, Veterinary Medical Teaching Hospital, Konkuk University, Seoul 05029, Korea \\ ${ }^{2}$ Department of Veterinary Surgery, College of Veterinary Medicine, Konkuk University, Seoul 05029, Korea
}

\begin{abstract}
Five paraplegic dogs were diagnosed with thoracolumbar intervertebral disc disease with more than $50 \%$ compression of spinal cord. Because the lesions were determined to be disc extrusion on magnetic resonance imaging, a hemilaminectomy was initially performed, however, protruded discs were confirmed during surgery. To remove the protruded disc, modified partial lateral corpectomy (mini-PLC) was additionally performed. All dogs recovered to full ambulation within a median of 44 days without temporary deterioration or vertebral instability. Mini-PLC as described here enables successful removal of the protruded disc, while preserving vertebral stability in dogs for whom the use of hemilaminectomy is inevitable.
\end{abstract}

Keywords: hemilaminectomy, mini-partial lateral corpectomy, paraplegic dogs, protruded disc, thoracolumbar intervertebral disc

*Corresponding author

Hun-young Yoon

Department of Veterinary Surgery, College of Veterinary Medicine, Konkuk University, 120, Neungdong-ro, Gwangin-gu, Seoul 05029, Korea

Tel: $+82-2-450-0494$

Fax: +82-2-444-4396

E-mail: yoonh@konkuk.ac.kr

ORCID

Hyun-Jung Han

https://orcid.org/0000-0002-1910-1261

Hun-Young Yoon

https://orcid.org/0000-0001-8834-952X

Conflict of Interest

The authors declare no conflicts of interest.

Received: May 27, 2019

Revised: August 25, 2019

Accepted: October 17, 2019
Partial lateral corpectomy (PLC) is defined as the partial removal of thoracic or lumbar adjacent vertebral bodies that support the herniated disc material inside the vertebral canal; this has appeared to be the most effective technique to treat protrusive thoracolumbar intervertebral disc disease (IVDD) in dogs, based on prior studies [1-3]. This technique has led to improved prognosis, relative to that achieved by any other surgical technique, in dogs with protrusive thoracolumbar IVDD, such that $90 \%$ of those undergoing PLC showed favorable outcomes with satisfactory spinal cord decompression [4]. In a certain situation, combined use of PLC and hemilaminectomy may be inevitable even though this combination has not been recommended because it could induce substantial vertebral instability or permanent intervertebral disc (IVD) space collapse, due to destruction of both lamina and vertebral body $[5,6]$.

This case report describes the use of mini-PLC, which was designed to markedly reduce the destruction associated with traditional PLC, for combined use with hemilaminectomy in five small dogs with thoracolumbar disc protrusion. To the best of our knowledge, this is the first report of this surgical technique for the treatment of heavily protrusive thoracolumbar IVDD in small dogs.

The present report includes five toy breed dogs: four Maltese and one Toy Poodle. The median age at diagnosis was 11 years (range, 7 to 12 years), and the median body weight was $3.8 \mathrm{~kg}$ (range, 2.5 to $9.6 \mathrm{~kg}$ ). There were four castrated males and one spayed female. Four dogs showed acute onset of paraplegia without any known cause. The remaining dog showed chronic, progressive onset of hindlimb weakness over a period of 6 months, which progressed to paraparesis 15 days before admission. At the time of admission, all five dogs showed paraplegia; three exhibited intact deep pain, while deep pain perception was not clearly identified in the remaining two dogs. The median duration to surgery was 5 days (range, 2 days to 6 months). The dogs had been treated with conservative treatment prior to admission (e.g., nonsteroidal or steroidal anti-inflammatory drug, gabapentin, and acupuncture). The overall clinical data are summarized in Table 1.

The dogs were definitively diagnosed with thoracolumbar IVDD by using a 1.5-T magnetic resonance imaging (MRI) machine (Magnetom Essenza 1.5-T; 
Table 1. Overall clinical data for five paraplegic dogs with thoracolumbar disc protrusion

\begin{tabular}{clcllccr}
\hline \hline $\begin{array}{c}\text { Case } \\
\text { number }\end{array}$ & Breed & $\begin{array}{c}\text { Age } \\
(\mathrm{yr})\end{array}$ & $\begin{array}{l}\text { BW } \\
(\mathrm{kg})\end{array}$ & Sex & $\begin{array}{c}\text { Onset of } \\
\text { clinical signs }\end{array}$ & $\begin{array}{c}\text { Neurologic } \\
\text { grade }^{*}\end{array}$ & $\begin{array}{c}\text { Duration to } \\
\text { surgery (day) }\end{array}$ \\
\hline 1 & Toy poodle & 7 & 9.6 & $\mathrm{CM}$ & Acute & 5 & 2 \\
2 & Maltese & 12 & 4.95 & $\mathrm{CM}$ & Acute & 4 & 3 \\
3 & Maltese & 12 & 2.5 & $\mathrm{SF}$ & Acute & 4 & 10 \\
4 & Maltese & 11 & 2.85 & $\mathrm{CM}$ & Chronic & 4 & 180 \\
5 & Maltese & 9 & 3.8 & $\mathrm{CM}$ & Acute & 5 & 5 \\
\hline
\end{tabular}

BW, body weight; CM, castrated male; SF, spayed female. "Neurological status was graded as: $0=$ no spinal hyperesthesia or paresis (disease free), $1=$ spinal hyperesthesia but no paresis, $2=$ ambulatory paraparesis, $3=$ non-ambulatory paraparesis, $4=$ paraplegia, $5=$ paraplegia and loss of deep pain perception.
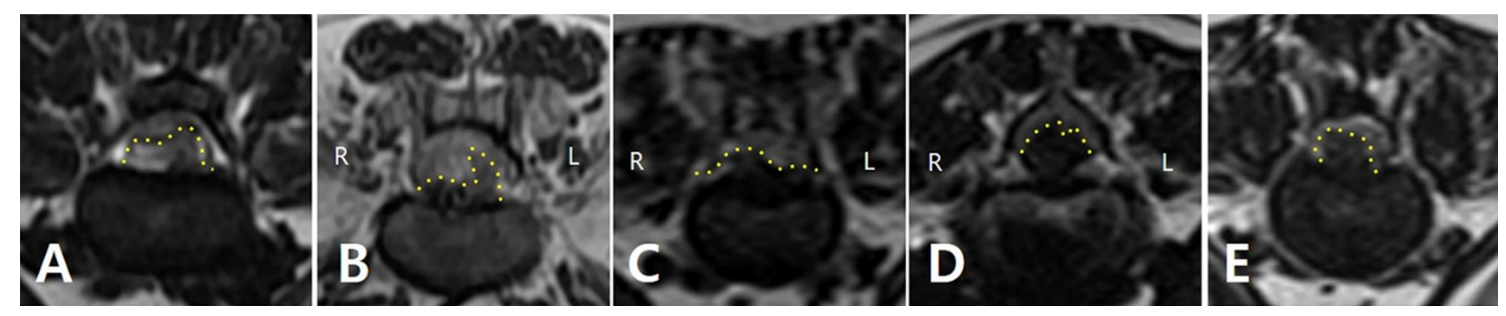

Fig. 1. Preoperative transverse T2-weighted magnetic resonance images. A large amount of disc material herniated centrally or laterally, leading to severe spinal cord compression over 50\% (yellow dotted line). (A) Case 1, 73\% compression at T13-L1, (B) Case 2, 50\% compression at L3-4, (C) Case 3, 50\% compression at T12-13, (D) Case 4, 80\% compression at T12-13, and (E) Case 5, 50\% compression at $\mathrm{T} 12-13$.

Table 2. Surgical site, proportion of slot height relative to vertebral body height, and postoperative outcomes in five paraplegic dogs with thoracolumbar disc protrusion treated by mini partial lateral corpectomy and hemilaminectomy

\begin{tabular}{|c|c|c|c|c|c|c|c|}
\hline \multirow{2}{*}{$\begin{array}{l}\text { Case } \\
\text { number }\end{array}$} & \multirow{2}{*}{$\begin{array}{c}\text { Surgical site } \\
\left.\text { (degree of compression }^{*}\right)\end{array}$} & \multirow{2}{*}{$\begin{array}{l}\mathrm{SH} \text { to } \mathrm{VBH} \\
\text { ratio }(\%)\end{array}$} & \multicolumn{3}{|c|}{ Recovery period (day) } & \multirow{2}{*}{ Complication } & \multirow{2}{*}{$\begin{array}{c}\text { Recurrence } \\
\text { (follow-up months) }\end{array}$} \\
\hline & & & $\mathrm{NG}^{\dagger} 4$ & $\mathrm{NG}^{\dagger} 2$ & $\mathrm{NG}^{\dagger} 0$ & & \\
\hline 1 & T13-L1 (73\%) & 18 & 1 & 4 & 45 & $\begin{array}{l}\text { Surgical wound } \\
\text { inflammation }\end{array}$ & None (16) \\
\hline 2 & L3-4 (50\%) & 25 & NA & 10 & 77 & Postoperative scoliosis & None (13) \\
\hline 3 & T11-12 (30\%), T12-13 (50\%) & 30.7 & NA & 3 & 18 & None & None (8) \\
\hline 4 & $\mathrm{~T} 12-13(80 \%)$ & 25 & NA & 14 & 44 & None & None (18) \\
\hline 5 & $\mathrm{~T} 12-13(50 \%)$ & 26.6 & 1 & 3 & 32 & None & None (4) \\
\hline
\end{tabular}

SH, slot height; VBH, vertebral body height; NG, neurologic grade; NA, not applicable. *Degree of preoperative spinal cord compression was calculated as a percentage of the degree of reduction of expected normal cross-sectional spinal cord area [6]; 'Neurological status was graded as: $0=$ no spinal hyperesthesia or paresis (disease free), $1=$ spinal hyperesthesia but no paresis, $2=$ ambulatory paraparesis, $3=$ nonambulatory paraparesis, $4=$ paraplegia, $5=$ paraplegia and loss of deep pain perception.

Siemens, Erlangen, Germany). MRI examinations revealed multiple IVDD in the dogs; all dogs had lesions with severe spinal cord compression of over $50 \%$, as follows: Case 1 , $73 \%$ compression at T13-L1; Case 2, 50\% compression at L3-4; Case 3, 50\% compression at T12-13; Case 4, 80\% compression at T12-13; and Case 5, 50\% compression at T12-13 (Fig. 1 and Table 2). The type of disc herniation was determined to be disc extrusion based on MRI findings such as the large volume or the lateralization of herniated disc material. Hyperintense intraparenchymal signal intensity changes around the compressed spinal cord locations were identified on sagittal T2-weighted images.

Considering the MRI findings, right- or left-sided hemilaminectomy was performed at T11-12, T12-13, T13-L1, or
L3-4 (Table 2). The dogs were premedicated with methylprednisolone sodium succinate (Predisol; Reyon Pharmaceutical, Korea) $15 \mathrm{mg} / \mathrm{kg} \mathrm{IV}$, cefazolin (Cefazoline inj.; Chong Kun Dang Parm, Korea) $20 \mathrm{mg} / \mathrm{kg}$ IV, butorphanol (Butophan inj.; Myungmoon Pharm, Korea) $0.2 \mathrm{mg} / \mathrm{kg} \mathrm{IV}$, famoti-

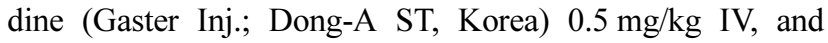
midazolam (Midazolam; Bukwang Pharmaceutical, Korea) 3 $\mathrm{mg} / \mathrm{kg}$ IV. Anesthesia was induced with propofol (Anepol Inj.; Hana Pharm, Korea) $4 \mathrm{mg} / \mathrm{kg}$ IV and maintained with isoflurane $(2 \%)$ in oxygen, following endotracheal intubation. Notably, extruded disc material was not identified through the hemilaminectomy window; however, the protruded disc was confirmed after careful manipulation of the spinal cord, in contrast to the MRI findings (Fig. 2A). To 

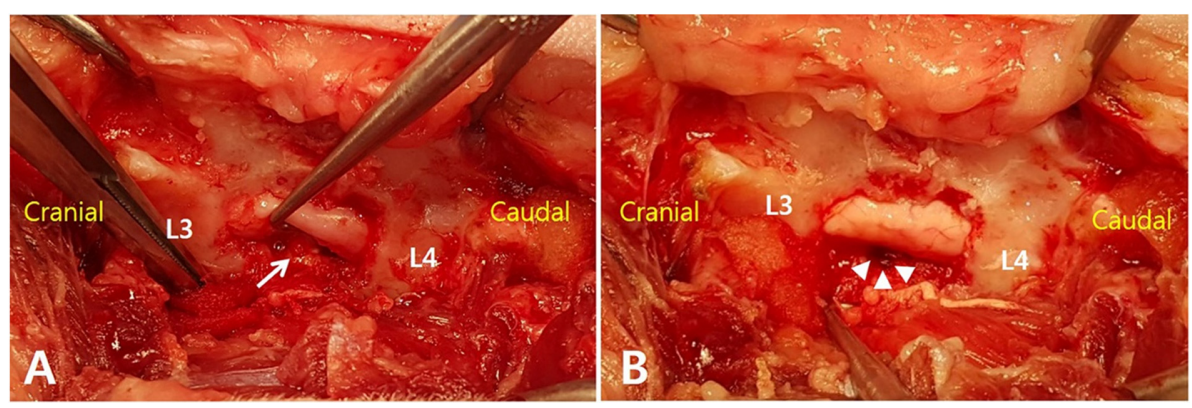

Fig. 2. Herniated disc material and spinal cord are investigated through the hemilaminectomy window: Left-sided hemilaminectomy at L3-4 in Case 2. (A) The protruded disc (white arrow) is confirmed via careful elevation of the spinal cord. (B) The protruded disc is completely removed with mini-partial lateral corpectomy (white arrowheads). The slot length depends on the craniocaudal margin of the protruded disc; the slot height was approximately $2 \mathrm{~mm}$. The spinal cord parenchyma appears normal without any injury or discoloration. L, lumbar vertebra.
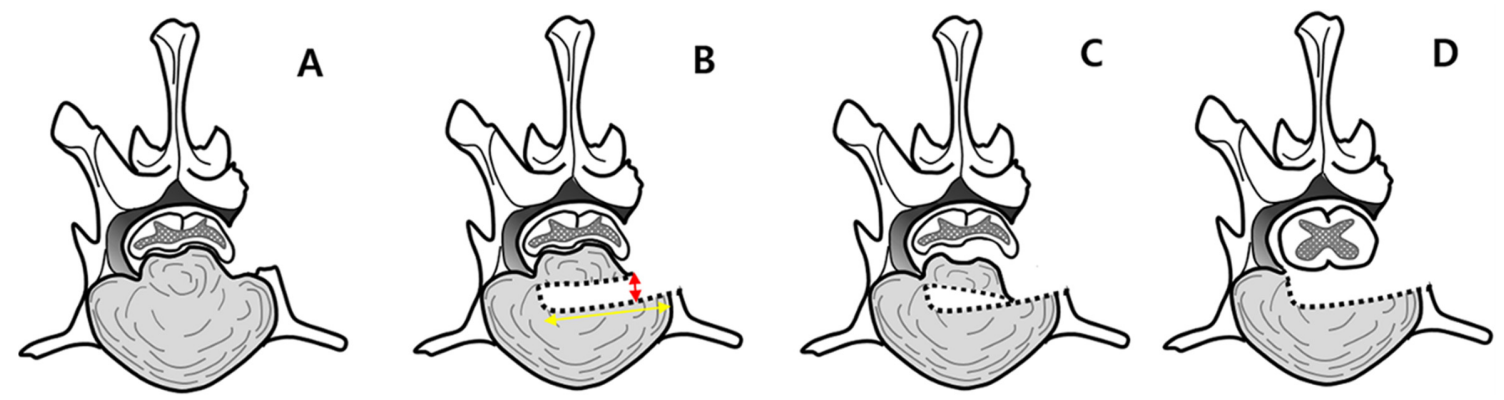

Fig. 3. Schematic diagram showing the process of mini-partial lateral corpectomy (PLC) performed after hemilaminectomy: transverse view of intervertebral disc space. (A) The intervertebral disc protrusion is identified through the hemilaminectomy window. (B) The slot of mini-PLC was made with approximately $2 \mathrm{~mm}$ from the bottom of the vertebral canal as slot height (red double arrow), and approximately $2 / 3$ of the vertebral body width as slot depth (yellow double arrow). (C) After creating the slot, the protruded disc is retracted into the slot, providing a space around the spinal cord, and is then scraped out with a curette. (D) The protruded disc is excised completely, and the compression of the spinal cord is resolved.

remove the protruded disc, mini-PLC was performed without additional vertebral exposure (Fig. 3). Before creating a slot, the location of the spinal nerve root was checked to prevent iatrogenic damage during the mini-PLC procedure. The slot was created using a round carbide burr with $1.6 \mathrm{~mm}$ head diameter attached to a high-speed pneumatic drill (Hall ${ }^{\circledR}$ MicroPower ${ }^{\mathrm{TM}}$ Orthopedic High Speed Drill, CONMED, USA). The anticipated slot dimensions of the mini-PLC were as follows: the slot depth was set as $2 / 3$ of the vertebral body's width, as described by Moissonnier et al. [3]; the slot length was adjusted based on craniocaudal extension of the protruded disc from the most cranial margin to the most caudal margin of the protruded disc; the slot height was $2 \mathrm{~mm}$ from the bottom of the vertebral canal to the ventral side. Vertebral body height at the vertebral end plate ranged from $6.5 \mathrm{~mm}$ to $11 \mathrm{~mm}$; the slot height of $2 \mathrm{~mm}$ corresponded to approximately 18 to $30 \%$ of the vertebral body height in all five dogs (Table 2). Drilling began at the center of the disc space, just beneath the bottom of the vertebral canal, then extended cranially and caudally in accordance with the anticipated slot height and length. The slot was then deepened in the laterolateral direction, parallel to the bottom of the vertebral canal and perpendicular to the mid-sagittal line of the vertebra in a horizontal plane, until the anticipated slot depth was achieved (Fig. 3B). This procedure was performed under saline irrigation to avoid thermal injury of adjacent structures. After creating the slot, the protruded disc was retracted into the level of the slot and removed by using a No. 11 scalpel blade, a spinal curette, and a rongeur while pressure was applied to the slot (Fig. 3C). The disc fragments were scraped out from the vertebral canal (Fig. 3D). Venous sinus hemorrhage was controlled with gelatin sponge (Hemospon; Technew, Brasil). After these procedures, the spinal cord was completely decompressed with no further herniated material (Fig. 2B). An autogenous fat graft was used to cover the exposed spinal cord and the surgical wound was closed in a routine fashion.

The dogs recovered from anesthesia without any complications. Postoperative care was identical for all dogs. For postoperative analgesia, a continuous rate infusion of fentanyl (Fentanyl Citrate Inj; Hana Pharm, Korea) 0.004 mg/kg/h and lidocaine (Daihan Lidocaine HCL Hydrate Inj.; Daihan 
Pharm, Korea) $1.2 \mathrm{mg} / \mathrm{kg} / \mathrm{h}$ was administered for $24 \mathrm{~h}$ after surgery, followed by oral carprofen (Rimadyl; Pfizer, USA) $2.2 \mathrm{mg} / \mathrm{kg} \mathrm{q} 12 \mathrm{~h}$ and tramadol (Tridol; Yuhan, Korea) $4 \mathrm{mg} /$ $\mathrm{kg} \mathrm{q} 12 \mathrm{~h}$ for 7 days. A cold pack was applied to the surgical wound for 3 days postoperatively, and rehabilitation (e.g., muscle massage, manipulation, and supported standing and walking) was applied gradually, based on the recovery status exhibited by each dog.

All dogs showed gradual postoperative improvement in their neurological status, such that they regained normal ambulation (Table 2). Two dogs without deep pain perception regained deep pain in both hindlimbs on the day after surgery. The five dogs were able to walk without assistance within a median of 4 days (range, 3 to 14 days) and recovered to full ambulation within a median of 44 days (range, 18 to 77 days). No dogs showed temporary deterioration of their neurological status after surgery; moreover, there were no changes in vertebral alignment, which would indicate instability of the vertebra, on postoperative radiographs. Two dogs showed minor postoperative complications, including surgical wound inflammation and temporary postoperative scoliosis; however, those complications resolved reasonably rapidly.

For the evaluation of long-term outcomes, the dogs were followed by using in-hospital physical examinations or by gathering information via telephone conversations with the owner over a period of 4 to 18 months. All dogs maintained normal ambulation without any recurrence of neurologic deficit or postoperative complications during the follow-up period.

Biomechanically, the IVD and articular facet are two major components responsible for vertebral stabilization: the IVD provides vertebral body stabilization by resisting flexion/ extension, lateral bending, and axial torsion; concomitantly, the articular facet plays an important role in vertebral lamina stabilization by resisting dorsal/ventral shear and torsion forces [6,7]. Thus, the combination of PLC and hemilaminectomy could induce clinically detrimental destabilization of both dorsal and ventral parts of the vertebra through simultaneous destruction of the vertebral body and articular facet $[5,6]$. This destabilization could lead to disastrous sequelae, such as vertebral subluxation and permanent IVD space collapse. Thus, current recommendations including avoiding the combination of hemilaminectomy with PLC $[1,5,6]$.

However, if a protruded disc is confirmed after hemilaminectomy has already been performed, as in the dogs in this study, PLC may inevitably be needed to successfully remove the protruded disc. This situation often occurs because an accurate preoperative determination of the type of IVD herniation is not possible before surgery, although proposed MRI guidelines have been developed and have improved the accuracy of differentiating extrusions and protrusions to $79.6 \%$ [8-10]. In the current study, hemilaminectomy was initially performed on the dogs because the results of the MRI examination indicated disc extrusion morphology, including lateralized disc material, narrowing of the IVD space, and subjective spinal cord swelling [9]. In particular, heavily herniated discs, such as those seen in the dogs in this report, tend to be initially assessed as disc extrusion, because the prevalence of compression of the spinal cord is reportedly significantly lower in dogs with disc protrusion (mean of $39 \%$ ) than in dogs with disc extrusion (mean of $50 \%$ ) [9]. Consequently, in contrast to the MRI findings, herniated discs were confirmed to be protrusion with or without extrusion, via hemilaminectomy in the dogs; thus, we decided to perform an additional PLC procedure, and to reduce the PLC slot size in order to preserve vertebral body stability.

For the mini-PLC, we attempted to preserve the slot depth within the range recommended in previous studies because it is the factor that most influences the degree of decompression, while slot height and craniocaudal length are reported to minimally affect the degree of decompression $[3,4]$. We easily adjusted the craniocaudal length of the slot according to the craniocaudal range of the protruded disc material, because the protruded disc was visually identified through the hemilaminectomy window [4]. The largest difference in slot dimensions from those of standard PLC was the slot height, which was significantly reduced in the current study. We decreased the slot height to $2 \mathrm{~mm}$, which was approximately $18-30 \%$ of the vertebral body height, compared to $50 \%$ of vertebral body height, as used in standard PLC $[3,4]$. Due to this considerable reduction of the slot height, we decreased the overall slot dimension by approximately 1.6fold to 2.7-fold; hence, we chose to describe this novel type of PLC as "mini-PLC." With this modification, we predicted that vertebral stability could be substantially increased by preserving approximately $70-82 \%$ of the vertebral body, despite destabilization of the vertebral lamina due to articular facet destruction. As a result, postoperative complications related to vertebral instability were not observed in any of the five dogs.

After the slot was made, the protruded disc was retracted towards the slot. Due to the reduced slot dimension, the protruded disc could not be completely retracted into the slot, so that additional removal of the remaining protruded disc was needed for complete decompression of the spinal cord. This procedure, so-called partial annulectomy, generally requires considerable manipulation of the spinal cord, which may cause iatrogenic spinal cord injury and a deterioration of postoperative neurologic status [3,4,11-13]. According to previous studies, successful outcomes as perceived by the operators were only $22 \%$ [12] to $30 \%$ [13] in dogs with disc protrusion undergoing hemilaminectomy and partial annulectomy, and $60 \%$ of the dogs showed deterioration of neurologic status within $24 \mathrm{~h}$ after surgery [12].

In the current study, the dogs did not show postoperative deterioration of neurological status and regained normal ambulation after the removal of the protruded disc. This excellent postoperative outcome was because the combined use of mini-PLC and hemilaminectomy provides sufficient space between the protruded disc and the compressed spinal 
cord to safely remove the protruded disc with minimal spinal manipulation. Moreover, through the hemilaminectomy window, we confirmed the structures of spinal cord, slot, and protruded disc accurately, leading to increased accessibility of the spinal cord and minimization of unnecessary manipulation.

There were no serious intraoperative complications in this study. Previous studies with PLC have noted a number of complications such as venous sinus hemorrhage (25\%), iatrogenic spinal nerve injury (5.6-8.3\%), and pneumothorax $(0.9 \%)[1,3,14]$. In this study, the spinal nerve root was wellvisualized through the hemilaminectomy window, thereby enabling iatrogenic damage to be avoided during the miniPLC procedure. In addition, although some venous sinus hemorrhage occurred, it was less severe than hemorrhage caused by laceration of the venous sinus due to drilling during standard PLC $[14,15]$. Moreover, hemostasis was rapidly accomplished because of easy access to the bleeding site and the placement of a hemostatic agent via the hemilaminectomy window. An additional advantage was that this technique did not require excessive handling of the rib head (i.e., removal or exarticulation), and did not involve the deep vertebral body approach that could cause pleural puncture and pneumothorax [4]. The low risk of such complications was similarly reported in a previous study in which mini-hemilaminectomy and PLC were used for large breed dogs with thoracolumbar IVDD, suggesting that the combination of PLC with other surgical procedures could reduce the complications of PLC [15]. This previous study reduced the complications associated combined use of PLC and hemilaminectomy by decreasing the extent of hemilaminectomy window and preserving the articular facet leading to facilitating the stabilization of the dorsal vertebral part $[6,15]$. In the present study, since hemilaminectomy was already performed, we reduced the PLC slot size for preserving more vertebral body than traditional PLC. Both modified techniques facilitated to preserve stability of the dorsal or ventral parts of vertebra in case of combined use of PLC and hemilaminectomy, leading to reduce the associated complications.

Limitations of our study included the small number of dogs and lack of long-term follow-up. Since this surgery was applied to only five dogs, further studies with larger populations should be undertaken to more fully evaluate the benefits of this combination of mini-PLC and hemilaminectomy.

The results of the present study suggest that when thoracolumbar disc protrusion is identified through hemilaminectomy, mini-PLC is a safe and effective treatment modality. Mini-PLC is a feasible alternative option for the successful removal of protruded discs, while preserving vertebral stability and reducing spinal cord manipulation, in dogs for whom the use of hemilaminectomy is inevitable.

\section{References}

1. Ferrand FX, Moissonnier P, Filleur A, Cachon T, Fau D,
Viguier E, Carozzo C. Thoracolumbar partial lateral corpectomy for the treatment of chronic intervertebral disc disease in 107 dogs. Ir Vet J 2015;68:27.

2. Jeffery ND, Harcourt-Brown TR, Barker AK, Levine JM. Choices and decisions in decompressive surgery for thoracolumbar intervertebral disc herniation. Vet Clin North Am Small Anim Pract 2018;48:169-186.

3. Moissonnier P, Meheust P, Carozzo C. Thoracolumbar lateral corpectomy for treatment of chronic disk herniation: technique description and use in 15 dogs. Vet Surg 2004;33:620-628.

4. Flegel T, Boettcher IC, Ludewig E, Kiefer I, Oechtering G, Böttcher P. Partial lateral corpectomy of the thoracolumbar spine in 51 dogs: assessment of slot morphometry and spinal cord decompression. Vet Surg 2011;40:14-21.

5. de Vicente F, Bernard F, FitzPatrick D, Moissonnier P. In vitro radiographic characteristics and biomechanical properties of the canine lumbar vertebral motion unit after lateral corpectomy, mini-hemilaminectomy and hemilaminectomy. Vet Comp Orthop Traumatol 2013;26:19-26.

6. Vizcaíno Revés N, Bürki A, Ferguson S, Geissbühler U, Stahl C, Forterre F. Influence of partial lateral corpectomy with and without hemilaminectomy on canine thoracolumbar stability: a biomechanical study. Vet Surg 2012;41:228-234.

7. Takeuchi T, Abumi K, Shono Y, Oda I, Kaneda K. Biomechanical role of the intervertebral disc and costovertebral joint in stability of the thoracic spine. A canine model study. Spine 1999;24:1414-1420.

8. De Decker S, Gomes SA, Packer RM, Kenny PJ, Beltran E, Parzefall B, Fenn J, Nair D, Nye G, Volk HA. Evaluation of magnetic resonance imaging guidelines for differentiation between thoracolumbar intervertebral disk extrusion and intervertebral disk protrusions in dogs. Vet Radiol Ultrasound 2016;57:526-533.

9. Gomes SA, Volk HA, Packer RM, Kenny PJ, Beltran E, De Decker S. Clinical and magnetic resonance imaging characteristics of thoracolumbar intervertebral disk extrusions and protrusions in large breed dogs. Vet Radiol Ultrasound 2016; 57:417-426.

10. Robertson I, Thrall DE. Imaging dogs with suspected disc herniation: pros and cons of myelography, computed tomography, and magnetic resonance. Vet Radiol Ultrasound 2011; 52 Suppl 1:S81-S84.

11. Coates JR. Intervertebral disk disease. Vet Clin North Am Small Anim Pract 2000;30:77-110.

12. Downes CJ, Gemmill TJ, Gibbons SE, McKee WM. Hemilaminectomy and vertebral stabilisation for the treatment of thoracolumbar disc protrusion in 28 dogs. J Small Anim Pract 2009;50:525-535.

13. Macias C, McKee WM, May C, Innes JF. Thoracolumbar disc disease in large dogs: a study of 99 cases. J Small Anim Pract 2002;43:439-446.

14. Salger F, Ziegler L, Böttcher IC, Oechtering G, Böttcher P, Flegel T. Neurologic outcome after thoracolumbar partial lateral corpectomy for intervertebral disc disease in $72 \mathrm{dogs}$. Vet Surg 2014;43:581-588.

15. Medl SC, Reese S, Medl NS. Individualized mini-hemilaminectomy-corpectomy (iMHC) for treatment of thoracolumbar intervertebral disc herniation in large breed dogs. Vet Surg 2017;46:422-432. 